\title{
ON THE EXISTENCE AND UNIQUENESS OF COMPLEX STRUCTURE AND SPACES WITH "FEW" OPERATORS ${ }^{1}$
}

\author{
BY
}

STANISLAW J. SZAREK

\begin{abstract}
We construct a $2 n$-dimensional real normed space whose (Banach-Mazur) distance to the set of spaces admitting complex structure is of order $n^{1 / 2}$, and two complex $n$-dimensional normed spaces which are isometric as real spaces, but whose complex Banach-Mazur distance is of order $n$. Both orders of magnitude are the largest possible. We also construct finite-dimensional spaces with the property that all "well-bounded" operators on them are "rather small" (in the sense of some ideal norm) perturbations of multiples of identity. We also state some "metatheorem", which can be used to produce spaces with various pathological properties.
\end{abstract}

1. Introduction and the main results. The main purpose of this paper is to prove the following results.

THEOREM 1.1. Given $n$ there exists a $2 n$-dimensional real normed space $X$ such that whenever $Y$ is an n-dimensional complex normed space and $Y_{\mathbf{R}}$ is $Y$, treated as a real space, then the Banach-Mazur distance $d\left(X, Y_{\mathbf{R}}\right) \geqslant c \sqrt{n}$, where $c$ is a numerical constant.

Given a complex (normed) space $Y$ denote by $\bar{Y}$ the space obtained from $Y$ by replacing the multiplication by scalar $(\lambda, y) \rightarrow \lambda y$ by $(\lambda, y) \rightarrow \lambda \odot y=\bar{\lambda} y$ and preserving the rest of the structure (addition, norm). We then have

THEOREM 1.2. Given $n$ there exists an n-dimensional space $Y$ over $\mathbf{C}$ such that $d(Y, \bar{Y}) \geqslant c n$, where $c$ is a numerical constant (here $d(\cdot, \cdot)$ denotes distance of complex spaces).

The orders of magnitude $O(\sqrt{n})$ and $O(n)$ in Theorems 1.1 and 1.2 are of course the largest possible: In the first case the $n$-dimensional (complex) Hilbert space always gives the upper estimate $\sqrt{2 n}$, in the second- $d\left(Y_{1}, Y_{2}\right) \leqslant n$ if $\operatorname{dim} Y_{1}=$ $\operatorname{dim} Y_{2}=n$ (in both the real and complex case). A weaker version of Theorem 1.1 (with estimate $O(\sqrt{n / \log n})$ ) was shown recently by P. Mankiewicz [8]; a version of Theorem 1.2 (with estimate $O(n / \log n)$ ) was shown, independently of this paper and approximately at the same time, by J. Bourgain [1], who also settled the infinite-dimensional problem, constructing a complex Banach space $Y$ such that $Y$ and $\bar{Y}$ are not isomorphic with all the consequences (see Corollary 1.3 below). Similar problems were considered recently by N. J. Kalton [5], who constructed an analogous example

Received by the editors November 1, 1984 and, in revised form, March 4, 1985.

1980 Mathematics Subject Classification. Primary 46B20, 51A99; Secondary 46L99, 47B10, 47D15, 52A22, 52A40, 60E15.

${ }^{1}$ Supported in part by NSF DMS- 8401906 . 
for quasi-Banach spaces and a Banach space $Y$ such that $Y$ and $\bar{Y}$ are not isometric. All the papers-except Kalton's-are heavily influenced by the "random" Gluskin's technique from [3] and some of the papers [4, 9].

Since $Y_{\mathbf{R}}$ and $\bar{Y}_{\mathbf{R}}$ (i.e., $Y$ and $\bar{Y}$ treated as real spaces) are obviously identical and the identity map is an isometry, we have

Corollary 1.3. Given $n$, there are two n-dimensional complex Banach spaces $Y_{1}, Y_{2}$ which are isometric as real spaces, but the complex Banach-Mazur distance $d\left(Y_{1}, Y_{2}\right)$ $\geqslant \mathrm{cn}$, where $\mathrm{c}$ is an absolute constant. Equivalently, there is a $2 n$-dimensional real Banach space admitting two complex structures, which differ (loosely speaking) in the worst imaginable way.

In the positive direction it should be noted that a real normed space $X$ admits complex structure (after some renorming) if e.g. $X$ is isomorphic to the direct sum $Z \oplus Z$ for some normed space $Z$, in particular if $X$ has symmetric (or subsymmetric) basis; one has also an obvious finite-dimensional version of this statement. One can, however, ask

Problem A. Does every space with unconditional basis (resp. Banach lattice) admit complex structure? The spaces constructed in this paper have very large unconditional basis constants (see [2]).

On the other hand, it is clear that if a complex space $Y$ has e.g. an unconditional basis, then $Y$ is isomorphic to $\bar{Y}$. However, it is not that clear that there is any (besides the Hilbert space) positive statement in the context of Corollary 1.3.

PROBlem B. Characterize the spaces which have unique (in the sense of Corollary 1.3) complex structure.

Theorems 1.1 and 1.2 will follow from the following "metatheorem", for which we need to introduce some notation. We will say that a linear operator $T: \mathbf{R}^{n} \rightarrow \mathbf{R}^{n}$ satisfies the condition $\left(M_{k, \alpha}\right)$ ( $M$ for "mixing") if

$$
\left(M_{k, \alpha}\right) \quad\left\{\begin{array}{l}
\text { there exists a subspace } H \subset \mathbf{R}^{n}, \operatorname{dim} H \geqslant k, \\
\text { such that }\left|P_{H^{\perp}} T_{\mid H}\right| \geqslant \alpha I_{H} .
\end{array}\right.
$$

Here $P_{H^{\perp}}$ denotes the orthogonal projection onto $H^{\perp}$, the absolute value and inequalities have the usual "Hilbert space" meaning; in this case the Hilbert space is just $l_{2}^{n}$ and the condition means that $\left\|P_{H^{\perp}} T x\right\|_{2} \geqslant \alpha\|x\|_{2}$ for $x \in H$ (here and later $\|\cdot\|_{p}$ denotes the usual $l_{p}^{n}$ norm).

THEOREM 1.4. Given $\delta>0$ there exists a norm $\|\cdot\|_{B}$ on $\mathbf{R}^{n},\|\cdot\|_{2} \leqslant\|\cdot\|_{B} \leqslant\|\cdot\|_{1}$, such that whenever $T$ satisfies $\left(M_{k, \alpha}\right)$ with some $k \geqslant \delta n$, then $\|T: B \rightarrow B\| \geqslant c \alpha \sqrt{n}$, where $c$ depends only on $\delta$. Moreover, one can require $\|\cdot\|_{B}$ to be invariant under some finite subgroup $\Gamma$ of $O(n)$, provided that $H$ in $\left(M_{k, \alpha}\right)$ can be chosen to be $\Gamma$-invariant; $c$ depends then additionally on the cardinality of $\Gamma$.

The following result strengthens Theorem 1.4 from [9] and is connected with the following well-known problem:

"Does there exist an infinite-dimensional Banach space such that every bounded operator on it is of the form $\lambda I+K$ with $K$ compact? (resp. K nuclear?)" 
First we need to introduce some notation. Given a nuclear operator $T: H \rightarrow \tilde{H}$ where ( $H, \tilde{H}$ are Hilbert spaces) define the quasi-norm

$$
\|T\|_{C_{0}}=\sum_{j} \frac{s_{j}(T)}{1+s_{j}(T)},
$$

where $\left(s_{j}(T)\right)$ is the sequence of $s$-numbers of $T$ (cf. $\S 2$ ). We then have

THEOREM 1.5. Given $\delta>0$ there exists $c=c(\delta)$ such that, for every $n \in \mathbf{N}$, there is a norm $\|\cdot\|_{B}$ on $\mathbf{R}^{n},\|\cdot\|_{2} \leqslant\|\cdot\|_{B} \leqslant\|\cdot\|_{1}$, with the property that if an operator $T$ satisfies $\|T: B \rightarrow B\| \leqslant c \sqrt{n}$, then there exists $\lambda \in \mathbf{R}$ such that $\|T-\lambda I\|_{C_{0}} \leqslant \delta n$. Moreover, one can choose $\|\cdot\|_{B}$ so that the cotype 2 constant of $B$ does not exceed $c_{1}$ and $B$ satisfies the Grothendieck theorem with constant $c_{1}\left(c_{1}\right.$ an absolute constant $)$; in particular $\pi_{1}\left(I: B \rightarrow l_{2}^{n}\right) \leqslant c_{1}$.

Theorems 1.4 and 1.5 (and 1.5A below) appear to be very useful facts. They reduce construction of spaces with some pathological properties to verifying simple conditions about operators on $\mathbf{R}^{n}$. Besides yielding other results of this paper they have several nontrivial known facts as easy corollaries. For example, the main results of [4 and 9] follow immediately from the obvious fact that if $P$ is a rank $k$ projection on $\mathbf{R}^{n}$, then $\|P-\lambda I\|_{C_{0}} \geqslant \frac{1}{3}[k \wedge(n-k)]$ combined with, respectively, Theorem 1.5 or $1.5 \mathrm{~A}$. Theorem 1.4 can be incorporated into the proof of the main result of [7]: the first part of [7] is devoted basically to showing a stronger version of our "mixing" condition for some group of operators which irreducibly act on $\mathbf{R}^{n}$; after this is proved, the rest follows from Theorem 1.4. Another possible application of Theorem 1.4 would be in constructing "poorly equivalent"-in the linear topological sense-representations of groups which are otherwise equivalent algebraically (Theorem 1.2 can be thought of as a result of this type for the circle group), etc.

We also have the following variants of Theorems 1.4 and 1.5.

THEOREM 1.4A. Given $n$ there exists a norm $\|\cdot\|_{B}$ on $\mathbf{R}^{n},\|\cdot\|_{2} \leqslant\|\cdot\|_{B} \leqslant\|\cdot\|_{1}$, such that whenever an operator $T$ satisfies the condition $\left(M_{k, \alpha}\right)$, then

$$
\|T: B \rightarrow B\| \geqslant c \alpha k / \sqrt{n(1+\ln n)},
$$

where $c$ is a universal constant. Moreover, $\|\cdot\|_{B}$ may be constructed to be invariant under the finite subgroup $\Gamma$ of $O(n)$ and $(O)$ still holds for $T$ 's satisfying $\left(M_{k, \alpha}\right)$ with $H$ invariant under $\Gamma$.

Note that $(O)$ is meaningful if $k / \sqrt{n(1+\ln n)}$ is large (e.g. $k \sim n^{\beta}, \beta>\frac{1}{2}$ ), a much weaker condition than $k \geqslant \delta n$ in Theorem 1.4. However, here we do not recover the sharp estimates on $\|T\|$ which we obtained in Theorem 1.4.

Let us introduce another quasi-norm defined for compact operators acting between Hilbert spaces:

$$
\|T\|_{w-C_{1}} \stackrel{\text { df }}{=}\left\|\left(s_{j}(T)\right)\right\|_{w-l_{1}}=\sup _{\lambda>0} \lambda \cdot \#\left\{j: s_{j}(T) \geqslant \lambda\right\}=\sup _{n \in \mathbf{N}} n \cdot s_{n}(T) .
$$


THEOREM 1.5A. There exists a norm $\|\cdot\|_{B}$ on $\mathbf{R}^{n},\|\cdot\|_{2} \leqslant\|\cdot\|_{B} \leqslant\|\cdot\|_{1}$, such that, for any linear operator $T$ on $\mathbf{R}^{n}$,

$$
\inf _{\lambda \in \mathbf{R}}\|T-\lambda I\|_{w-C_{1}} \leqslant c \sqrt{n(1+\ln n)}\|T: B \rightarrow B\|
$$

where $C$ is an absolute constant.

Since we obviously have $\|T\|_{C_{1}} \leqslant(1+\ln n)\|T\|_{w-C_{1}}\left(C_{1}\right.$ is the nuclear norm) for $T$ a rank $n$ operator, we have

Corollary 1.6. There exists a norm $\|\cdot\|_{B}$ on $\mathbf{R}^{n},\|\cdot\|_{2} \leqslant\|\cdot\|_{B} \leqslant\|\cdot\|_{1}$, such that, for any operator $T$ on $\mathbf{R}^{n}$,

$$
\inf _{\lambda \in \mathbf{R}}\|T-\lambda I\|_{C_{1}} \leqslant C \sqrt{n(1+\ln n)^{3}}\|T: B \rightarrow B\| .
$$

Theorems 1.5 and $1.5 \mathrm{~A}$ and Corollary 1.6, although clearly relevant to the problem stated before Theorem 1.5, have an obvious drawback: the norms of $T-\lambda I$, which appear in their statements, are not intrinsic to $B$. Instead, they come from some inner product norm (closely connected with $B$, though).

ACKNOWLEDGMENTS. A large part of this research was done while the author was in residence at the Banach Space Workshop at the Ohio State University in July 1984. The author would like to thank Gilles Pisier for communicating the problems (which led to Theorems 1.1 and 1.2) and the result of P. Mankiewicz.

2. Notation and organization of the paper. We use the standard Banach space notation as can be found e.g. in [6]. If $X$ is a (normed) linear space, $L(X)$ will denote the space of (bounded) linear operators on $X . L(X)$ will be endowed with the standard operator norm $\|\cdot\|_{L(X)}$, occasionally denoted by $\|\cdot: X \rightarrow X\|$ or $\|\cdot\|_{X \rightarrow X}$, although we may occasionally consider other operator (usually ideal) norms or quasi-norms. By $I_{X}$ we will denote the identity map on $X$; occasionally we will omit the subscript $X$. By $B(X)$ we will denote the unit ball of $X$. If $X=l_{p}^{n}$, we will write $B_{p}^{n}$ for $B\left(l_{p}^{n}\right)$. We will frequently identify a normed space $X$ with its unit ball $B(X)$ and its norm $\|\cdot\|_{X}$. For a set $F \subset X$ we will denote by ac $(F)$ its absolute convex hull and by $[F]$ its linear span.

All inner product spaces considered in this paper are de facto subspaces of $l_{2}^{n}$ for appropriate $n$ and so we will denote every inner product norm by $\|\cdot\|_{2}$. If $H$ is a Hilbert space and $E$ its subspace, we will denote by $P_{E}$ the orthogonal projection from $H$ onto $E$. If $T: H \rightarrow \tilde{H}$ ( $\tilde{H}$ also a Hilbert space) is compact, then $T$ admits the polar decomposition, i.e. can be written

$$
T=\sum_{j \geqslant 1} \lambda_{j}\left\langle u_{j}, \cdot\right\rangle \tilde{u}_{j},
$$

where $\lambda_{1} \geqslant \lambda_{2} \geqslant \cdots>0$ and $\left(u_{j}\right)$ and $\left(\tilde{u}_{j}\right)$ are orthonormal sequences in $H$ and $\tilde{H}$ respectively. The representation (PD) is "nearly" unique; certainly the sequence $\left(\lambda_{j}\right)$ is unique and so are (up to a sign) $\left(u_{j}\right)$ and $\left(\tilde{u}_{j}\right)$ if all $\lambda_{j}$ 's are distinct. $\lambda_{j}$ 's are sometimes called the $s$-numbers of $T$ and denoted by $s_{j}(T)$. Another description of $s$-numbers involves the concept of a modulus of an operator defined by $|T|=$ $\left(T^{*} T\right)^{1 / 2} ; \quad\left(s_{j}(T)\right)$ is then the sequence of eigenvalues of $T$ arranged in the nonincreasing order, $u_{j}$ 's are corresponding (normalized) eigenvectors. 
We will work with the usual order on $L(H)$. For our purposes it is enough to know that if $S T=T S$, then $|S| \geqslant|T|$ iff $\|S x\|_{2} \geqslant\|T x\|_{2}$ for all $x \in H$. Also, let us state explicitly some immediate consequence of the existence of (PD): if $\operatorname{dim} H=n$, $\alpha \geqslant 0$ and $1 \leqslant k \leqslant n$, then either there exists a subspace $E \subset H, \operatorname{dim} E \geqslant k$, such that $\left|T_{\mid E}\right| \leqslant \alpha I_{E}$ or a subspace $F \subset H, \operatorname{dim} F>n-k$, such that $\left|T_{\mid F}\right|>\alpha I_{F}$.

We will consider the trace classes $C_{p} \subset L(H)$ with norm $\|T\|_{C_{p}}=\left(\operatorname{tr}|T|^{p}\right)^{1 / p}=$ $\left\|s_{j}(T)\right\|_{p}$ (in particular the nuclear norm $C_{1}$ ) and some unitary ideal quasi-norms corresponding to various symmetric quasi-norms on $\mathbf{R}^{n}$ :

$$
\begin{gathered}
\|T\|_{C_{0}} \stackrel{\mathrm{df}}{=} \sum_{j} s_{j}(t) /\left(1+s_{j}(t)\right) \\
\|T\|_{w-C_{1}}=\left\|\left(s_{j}(t)\right)\right\|_{w-l_{1}}=\sup _{\lambda>0} \lambda \cdot\left\{j: s_{j}(T) \geqslant \lambda\right\}=\sup _{j} j s_{j}(T) .
\end{gathered}
$$

It should be noted that $\|\cdot\|_{C_{0}}$ satisfies the triangle inequality, but is not positively homogeneous; $\|\cdot\|_{w-C_{1}}$ is positively homogeneous, but satisfies just $\|S+T\|_{w-C_{1}}$ $\leqslant 2\left(\|S\|_{w-C_{1}}+\|T\|_{w-C_{1}}\right)$.

If $\operatorname{dim} H=m$, we will say that a ( $H$-valued) random variable (r.v.) $g$, defined on some probability space $(\Omega, \Sigma, P)$, has the (Gaussian) distribution $N\left(0, \sigma^{2}, H\right)$ if its density equals $\left(m / 2 \pi \sigma^{2}\right)^{m / 2} e^{-m\|x\|_{2}^{2} / 2 \sigma^{2}}$. We emphasize that, in our normalization, $E\|g\|_{2}^{2}=\sigma^{2}$. We will need just three facts about such $g$ :

(i) $\mathscr{P}\left(\left\{\|g\|_{2} \geqslant 2 \sigma\right\}\right) \leqslant \alpha^{n}, \alpha<1$ a numerical constant.

(ii) if $E \subset H, \operatorname{dim} E=k$, then $P_{E} g$ has the distribution $N\left(0, k \sigma^{2} / m, E\right)$.

(iii) if $E, F \subset H, E \perp F$, then $P_{E} g$ and $P_{F} g$ are independent.

Beginning with $\S 4$, all spaces considered in this paper are real; in $\S \S 1$ and 3 it is always carefully stated whether we deal with the real or complex case.

The paper is organized as follows.

$\S 1$ presents the main results: Theorems 1.1,1.2,1.4 and 1.5 together with their corollaries and generalizations, comments and some open problems.

$\$ 3$ contains reduction of Theorems 1.1 and 1.2 to statements about real spaces: Propositions 3.1 and 3.2. The corresponding "mixing properties" (Lemmas 3.4 and 3.5), from which the propositions follow via Theorem 1.4, are also stated there.

In $\$ 4$ the "mixing" Lemmas 3.4 and 3.5 are proved.

$\$ 5$ contains the proof of Theorem 1.4 and a sketch of the proof of its generalization-Theorem 1.4A.

In $\S 6$ Theorems 1.5 and $1.5 \mathrm{~A}$ are deduced from Theorems 1.4 and $1.4 \mathrm{~A}$ respectively.

3. Reduction to problems about real spaces. If $X$ is a normed space over $\mathbf{R}$, then any complex structure on $X$ (i.e. multiplication elements of $X$ by complex scalars) corresponds (on an essentially one-to-one basis) to the R-linear isometry on $X$ such that $A^{2}=-I$. Indeed, if multiplication by complex scalars satisfying $\|\lambda x\|=|\lambda| \cdot\|x\|$ is defined, set $A x=i x$. Conversely, if such an isometry exists, define $i x=A x$ or, more generally, $(a+b i) x=a x+b A x$, to get that $\|(a+b i) x\|=|a+b i| \cdot\|x\|$ one 
must modify the norm a little bit, introducing e.g.

$$
\|x\|^{1 \stackrel{\mathrm{df}}{=}} \frac{1}{2 \pi} \int_{0}^{2 \pi}\|(\cos \theta) x+(\sin \theta) A x\| d \theta
$$

clearly $\|\cdot\|^{1} \widetilde{\sqrt{2}}\|\cdot\|$.

Taking this into account, Theorem 1.1 immediately reduces to

Proposition 3.1. Given $n$ there exists a $2 n$-dimensional real normed space $X$ such that whenever $S: X \rightarrow X$ satisfies $S^{2}=-I$, then $\|S\| \geqslant c_{1} \sqrt{n} ; c_{1}$ a numerical constant.

To reformulate Theorem 1.2 notice that if $Y$ is a complex space and $A$ is the isometry associated with the regular multiplication (i.e., $A x=i x$ ), then the isometry determining the complex structure of $\bar{Y}$ is $-A$, as $i \odot x=-i x=-A x$. Therefore to say that an R-linear operator $T: Y_{\mathbf{R}} \rightarrow Y_{\mathbf{R}}$ is $\mathbf{C}$-linear considered as acting from $Y$ to $\bar{Y}$ (i.e., $T(i x)=i \odot T x$ ) is precisely the same as to say that $T A=-A T$. Thus Theorem 1.2 reduces to

Proposition 3.2. Given $n$ there exist a $2 n$-dimensional real space $X$ and an isometry $A$ on $X$ satisfying $A^{2}=-I$ such that whenever $T: X \rightarrow X$ satisfies $T A=-A T$, then $\|T\| \cdot\left\|T^{-1}\right\| \geqslant c_{1} n, c_{1}$ a numerical constant.

For future use observe that $T: Y_{\mathbf{R}} \rightarrow Y_{\mathbf{R}}$ is $\mathbf{C}$-linear considered as an operator acting on $Y$ (or $\bar{Y}$ ) iff $T A=A T$.

For the rest of this section and in $\$ 4$, all normed spaces will be "living" on $\mathbf{R}^{2 n}$; they will then be determined by (and frequently identified with) their unit balls. We will work with the standard $l_{2}^{2 n}$ Hilbert space structure.

REMARK 3.3. When working with the product $\|T\| \cdot\left\|T^{-1}\right\|$ we are always free to replace $T$ by any $a T, a \in \mathbf{R} \backslash\{0\}$, without affecting the value of the product. For the purpose of the proof of Proposition 3.2 we will use a normalization such that both $T$ and $T^{-1}$ have at least $n s$-numbers greater than or equal to 1 ; in other words, if $T=\sum_{j=1}^{2 n} \sigma_{j}\left(u_{j}, \cdot\right) v_{j}$ is the polar decomposition of $T$, then $\sigma_{1} \geqslant \sigma_{2} \geqslant \cdots$ $\geqslant \sigma_{n} \geqslant 1 \geqslant \sigma_{n+1} \geqslant \cdots \geqslant \sigma_{2 n}$.

Both Propositions 3.1 and 3.2 assert that norms of certain operators are large. It is therefore clear that the propositions can be deduced via Theorem 1.4 if we show that those operators satisfy certain "mixing properties", which we now formulate.

LEMMA 3.4. If $S: l_{2}^{2 n} \rightarrow l_{2}^{2 n}$ is such that $S^{2}=-I$, then it satisfies the "mixing property" $\left(M_{n, 1}\right)$.

LEMMA 3.5. Let $A \in O(2 n)$ be such that $A^{2}=-I$ and let $T: l_{2}^{2 n} \rightarrow l_{2}^{2 n}$ verify

(i) $A T=-T A$,

(ii) at least $n$ s-numbers of $T$ are greater than or equal to 1 (cf. Remark 3.3).

Then $T$ satisfies the "mixing property" $\left(M_{m, 1}\right)$ with $m \geqslant n / 8-1$ and $H$ such that $A H=H$.

Assuming the lemmas above (to be proved in \$4) it is immediate to deduce Propositions 3.1 and 3.2 (and hence Theorems 1.1 and 1.2) from Theorem 1.4 (proved in §5). Indeed, to prove Proposition 3.1, we apply the first part of Theorem 
1.4 with $2 n$ instead of $n, \delta=1 / 2, \alpha=1$. To prove Proposition 3.2, we apply the second part of Theorem 1.4 with e.g. $\delta=1 / 17, \alpha=1$ and $\Gamma=\{I, A,-I,-A\}$ (cf. Remark 3.3).

4. The "mixing" properties. In this section we prove Lemmas 3.4 and 3.5. Let us reformulate slightly the first of them.

LEMMA 3.4A. If $S: l_{2}^{2 n} \rightarrow l_{2}^{2 n}$ is such that $S^{2}=-I$, then there is $H \subset l_{2}^{2 n}$, $\operatorname{dim} H=n$, such that, for $x \in H$,

$$
\left\|P_{H^{\perp}} S x\right\|_{2}=\|S x\|_{2} \geqslant\|x\|_{2} \text {. }
$$

Proof. Consider the polar decomposition $S=\sum_{j=1}^{2 n} \lambda_{j}\left(u_{j}, \cdot\right) v_{j}$ (with $\lambda_{j}>0,\left(\lambda_{j}\right)$ nondecreasing). Since $S=-S^{-1}$, we also have $S=-\sum_{j=1}^{2 n} \lambda_{j}^{-1}\left(v_{j}, \cdot\right) u_{j}$. By the uniqueness properties of polar decomposition, it follows that $\lambda_{j}=\lambda_{2 n-j+1}^{-1}$ for all $j$ (in particular $\lambda_{n+1}=\lambda_{n}^{-1} \leqslant 1 \leqslant \lambda_{n}$ and hence $\lambda_{j} \geqslant 1$ for $j \leqslant n$ ). If all $\lambda_{j}$ 's were distinct, it would also immediately follow that $v_{j}= \pm u_{2 n-j+1}$; in the general case we can also achieve that by modifying the original $u_{j}$ 's and $v_{j}$ 's somewhat. Thus we can write

$$
S=\sum_{j=1}^{n} \lambda_{j}\left(u_{j}, \cdot\right) v_{j}-\sum_{j=1}^{n} \lambda_{j}^{-1}\left(v_{j}, \cdot\right) u_{j},
$$

where $\left(u_{1}, u_{2}, \ldots, u_{n}, v_{1}, \ldots, v_{n}\right)$ is an orthonormal basis of $l_{2}^{2 n}$. Let $H=\left[u_{j}\right]_{j \leqslant n}$. Then $H^{\perp}=\left[v_{j}\right]_{j \leqslant n}$ and

$$
P_{H^{\perp}} S=\sum_{j=1}^{n} \lambda_{j}\left(u_{j}, \cdot\right) v_{j} .
$$

Since all $\lambda_{j}$ 's, $1 \leqslant j \leqslant n$, are greater than or equal to 1 , the assertion of Lemma 3.4A readily follows.

The proof of Lemma 3.5 is a little bit more complicated. We shall work with $A$ whose matrix representation in the standard unit vector basis $\left(e_{1}, \ldots, e_{n}\right.$, $\left.e_{n+1}, \ldots, e_{2 n}\right)$ of $\mathbf{R}^{2 n}$ is

$$
\left[\begin{array}{cc}
0 & -I \\
I & 0
\end{array}\right]
$$

where the entries stand for $n \times n$ matrices (i.e. $A e_{j}=e_{n+j}$ and $A e_{n+j}=-e_{j}$ for $j=1,2, \ldots, n)$. This can always be achieved by appropriate change of coordinates-orthogonal, if $A \in O(2 n)$ (see the proof of Lemma 3.4). Actually for Proposition 3.2 we just need to find some $A$ and $X$, so we could as well start with $A$ of the form (1).

Let us reformulate the lemma.

LEMMA 3.5A. Let $A$ be given by (1) and let $T: l_{2}^{2 n} \rightarrow l_{2}^{2 n}$ satisfy

(i) $A T=-T A$,

(ii) at least $r$-numbers of $T$ are $\geqslant 1$.

Then there exist $H \subset l_{2}^{2 n}, \operatorname{dim} H \geqslant r / 8-1, A H=H$, such that, for $x \in H$,

$$
\left\|P_{H^{\perp}} T x\right\|_{2}=\|T x\|_{2} \geqslant\|x\|_{2} .
$$

For the proof of Lemma 3.5A we need several further lemmas. 
LemmA 4.1. If $A: l_{2}^{2 n} \rightarrow l_{2}^{2 n}$ is given by (1) and $T: l_{2}^{2 n} \rightarrow l_{2}^{2 n}$ satisfies $T A=-A T$, then there exists an orthonormal sequence $h_{1}, h_{2}, \ldots, h_{k}$ with $k \geqslant n / 4$ such that one has

(j) $E_{i} \perp E_{j}$ for $i \neq j$, where $E_{j}=\left[h_{j}, A h_{j}, T h_{j}, T A h_{j}\right]$ for $j=1,2, \ldots, k$,

(jj) $\left\|T h_{j}\right\|_{2} \geqslant S_{8(j-1)+1}(T)$ for $j=1,2, \ldots, k$.

Proof. Set $E_{0}=\{0\}$ and let $k$ be the smallest integer greater than or equal to $n / 4$. For $j=1,2, \ldots, k$ choose

$$
h_{j} \in\left(E_{1}+\cdots+E_{j-1}\right)^{\perp} \cap T^{-1}\left(\left(E_{1}+\cdots+E_{j-1}\right)^{\perp}\right) \stackrel{\text { df }}{=} E
$$

such that $\left\|h_{j}\right\|_{2}=1$ and $\left\|T h_{j}\right\|_{2} \geqslant S_{8(j-1)+1}(T)$; this is possible since codim $E \leqslant$ 8(j-1). Obviously, $h_{j} \perp E_{i}$ and $T h_{j} \perp E_{i}$ for $i<j$. Also $A h_{j} \perp A E_{i}=E_{i}$ and $T A h_{j}=-A T h_{j} \perp A E_{i}=E_{i}$ for $i<j$. So $E_{j} \perp E_{i}$ for $i<j$.

Q.E.D.

LEMMA 4.2. If $S: H \rightarrow H$ ( $H$ a Hilbert space $)$ and $u, u^{\prime} \in H \backslash\{0\}$ satisfy $(S u, u)\left(S u^{\prime}, u^{\prime}\right) \leqslant 0$, then there exists $w \in\left[u, u^{\prime}\right],\|w\|_{2}=1$, such that $(S w, w)=0$.

Proof. Apply the Intermediate Value Theorem to the function $[0, \pi / 2] \ni \theta \rightarrow$ $\left(S w_{\theta}, w_{\theta}\right)$, where $w_{\theta}=u \cdot \cos \theta+u^{\prime} \cdot \sin \theta$.

Q.E.D.

LEMMA 4.3. If $A$ and $T$ are as in Lemma 4.1 and $h \in l_{2}^{2 n}$ with $\|h\|_{2}=1$, then there exist $u, v \in[h, A h]$ such that $A u=v,\|u\|_{2}=\|v\|_{2}=1$ and $(T u, v)=(T v, u)=0$. Moreover, $(u, v)=0,(T u, u)=-(T v, v)$ and $\|T u\|_{2}=\|T v\|_{2}=\|T h\|_{2}$.

Proof. Notice first that $(A x, x)=0$ for every $x \in l_{2}^{2 n}$. Since $(h, A h)=(T h, T A h)$ $=0$ and $\|T h\|_{2}=\|T A h\|_{2}$, it easily follows that $\|T x\|_{2}=\|T h\|_{2}$ for every $x \in[h, A h]$ with $\|x\|_{2}=1$. The fact that one can choose $u \in[h, A h]$ with $\|u\|_{2}=1$ such that if $v=A u$, then $(T v, u)=(T A u, u)=0$ follows from Lemma 4.2 applied with $S=T A$, $u=h, u^{\prime}=A h$. All other statements follow trivially from these and the assumptions.

Q.E.D.

Proof of Lemma 3.5A. Let $h_{1}, h_{2}, \ldots, h_{k}$, with $k \geqslant n / 4$, be an orthonormal sequence constructed as in Lemma 4.1. For every $i$, find $u_{i}, v_{i} \in\left[h_{i}, A h_{i}\right]$ as in Lemma 4.3; then $v_{i}=A u_{i}$ and $\left(u_{i}, v_{i}\right)=\left(T u_{i}, v_{i}\right)=\left(T v_{i}, u_{i}\right)=0$. Also $\left\|T u_{i}\right\|_{2}=$ $\left\|T v_{i}\right\|_{2}=\left\|T h_{i}\right\|_{2} \geqslant 1$ for $i \leqslant d$, where $d \geqslant r / 8$ (by (ii) of Lemma 3.5A and (jj) of Lemma 4.1). Therefore

$$
T u_{i}=a_{i} u_{i}+z_{i}, \quad T v_{i}=-A T u_{i}=-a_{i} v_{i}-A z_{i}
$$

with $z_{i}, A z_{i} \in\left[u_{i}, v_{i}\right]$ and $\left(z_{i}, A z_{i}\right)=0$. In particular

$$
\left[h_{i}, A h_{i}, T h_{i}, T A h_{i}\right]=E_{i}=\left[u_{i}, v_{i}, z_{i}, A z_{i}\right] .
$$

Exchanging the roles of $u_{i}$ 's and $v_{i}$ 's for some $i$ 's, if necessary, we may assume that the sequence $\left(a_{i}\right)$ has alternating signs. For $j=1,2, \ldots,[d / 2]$, apply Lemma 4.2 with $\left\{u, u^{\prime}\right\}=\left\{u_{2 j-1}, u_{2 j}\right\}$ to obtain an orthonormal sequence $\left(w_{j}\right)$ with $w_{j} \in\left[u_{2 j-1}, u_{2 j}\right] \subset E_{2 j-1} \oplus E_{2 j}$ and $\left(T w_{j}, w_{j}\right)=0$. Since $T w_{j} \in E_{2 j-1} \oplus E_{2 j}$, we also have $\left(T w_{j}, w_{i}\right)=0$ for $i \neq j$.

Let $H_{0}=\left[w_{j}\right]_{j \leqslant[d / 2]}$ and let $H=H_{0}+A H_{0}$. We already know that $T w_{j} \perp H_{0}$ for $j \leqslant[d / 2]$. Since $T w_{j} \in\left[u_{2 j-1}, u_{2 j}, z_{2 j-1}, z_{2 j}\right] \perp\left[v_{2 i}, v_{2 i-1}\right] \ni A w_{i}$ for $i, j \leqslant$ [d/2], also $T w_{j} \perp A H_{0}$. So $T w_{j} \perp H$ and hence $T H_{0} \perp H$. It follows in particular 
that $T A H_{0}=-A T H_{0} \perp A H=H$. Thus $T H \perp H$. Since

$$
\left\|T w_{j}\right\|_{2} \geqslant \min \left\{\left\|T u_{2 j-1}\right\|_{2},\left\|T u_{2 j}\right\|_{2}\right\} \geqslant 1
$$

and the $T w_{j}$ 's are mutually orthogonal, it follows that $\left\|P_{H^{\perp}} T x\right\|_{2}=\|T x\|_{2} \geqslant\|x\|_{2}$ for $x \in H$. This proves Lemma 3.5A.

Q.E.D.

5. Proof of Theorem 1.4. The proof of Theorem 1.4 consists of three parts: (a) description of the random norm $\|\cdot\|_{B}$ on $\mathbf{R}^{n}$, (b) the "measure theoretic" argument which shows that, for a given operator $T$ satisfying the appropriate "mixing condition", $\|T: B \rightarrow B\|$ is large for the vast majority of $B$ 's and (c) the " $\varepsilon$-net" argument which shows that, still for "most of" $B$ 's, $\|T: B \rightarrow B\|$ is large for all such $T$ 's.

We shall prove Theorem 1.4 only in the case relevant to Proposition 3.2 (and Theorem 1.2), i.e. $\Gamma=\{I, A,-I,-A\}$ with $A$ of the form (1) (see $\$ 4$ ), which reveals all technical difficulties. The case of trivial $\Gamma=\{I,-I\}$ relevant to Proposition 3.1 (and Theorem 1.1) is somewhat easier and can be obtained from the argument we present by, roughly speaking, dropping all references to $A$ and expressions involving $A$; the case of general $\Gamma$ requires "throwing in" additional expressions involving all elements of $\Gamma$ different from $I$. We point out the crucial observation that if $G_{0} \subset \mathbf{R}^{n}$ then there is $G \supset G_{0}$ which is $\Gamma$-invariant with $\operatorname{dim} G \leqslant \# \Gamma \cdot \operatorname{dim} G_{0}$.

(a) Description of the random norm. Fix $\delta>0$ and let $k \geqslant \delta n$. Let $m \leqslant k / 10$ and let $g_{1}, g_{2}, \ldots, g_{m}$ be independent Gaussian random variables (r.v.'s) with distribution $N\left(0,1, l_{2}^{n}\right)$ defined on some probability space $(\Omega, \Sigma, \mathscr{P})$. For each $\omega \in \Omega$ denote $B=B(\omega)=\operatorname{ac}\left\{e_{1}, e_{2}, \ldots, e_{n}, g_{1}, \ldots, g_{m}, A g_{1}, \ldots, A g_{m}\right\}$. We shall identify $B$ with the normed space $\left(\mathbf{R}^{n},\|\cdot\|_{B}\right)$ whose unit ball is $B$. Note that $A(B)=B$ and hence $\|x\|_{B}=\|A x\|_{B}$ for $x \in \mathbf{R}^{n}$, i.e. $\|\cdot\|_{B}$ is $\Gamma$-invariant. Let us observe here (this is going to be useful in the proof of Theorem 1.5) that every space constructed in this way is a quotient of $l_{1}^{N}$ with $N \leqslant 2 n$. Moreover, the randomness in the construction allows us to conclude that, with large probability, the kernel of the projection (and in fact also the orthogonal-in $l_{2}^{N}$-complement of the kernel) are "nearly" Euclidean subspaces of $l_{1}^{N}$. In the case of trivial $\Gamma$ (i.e. $B=\operatorname{ac}\left\{e_{1}, \ldots, e_{n}, g_{1}, \ldots, g_{m}\right\}$ ) this follows directly from [9, Remark 4.5]; the "complex version" of the argument presented there also works if $\Gamma=\{I, A,-I,-A\}$, i.e. the case we describe here in detail (we do not make any claim about the kernel in the case of general $\Gamma$ ). In particular it will follow that all spaces, whose existence is asserted in Theorems 1.1,1.2, 1.5, and the cases of Theorem 1.4 relevant to the $\Gamma$ 's mentioned above can be assumed to be quotients of $l_{1}^{N}$ by a "nearly" Euclidean subspace with all the consequences like having cotype 2 constant bounded by a universal constant $c_{1}$ and satisfying the Grothendieck theorem with the constant $c_{1}$ (see [9, Remark 4.5] and its references).

(b) The "measure theoretic" argument. We will consider operators $T$ satisfying the condition $\left(M_{k, \alpha}\right), k \geqslant \delta n$. Clearly it is enough to work with $\alpha=1$, i.e. with the condition

$$
\left\{\begin{array}{l}
\text { there exists } H \subset \mathbf{R}^{n}, \operatorname{dim} H \geqslant k, \text { such that } \\
A H=H \text { and }\left\|P_{H^{\perp}} T x\right\|_{2} \geqslant\|x\|_{2} \text { for } x \in H .
\end{array}\right.
$$


For technical reasons we will also use a slightly weaker form of (2):

$$
\left\{\begin{array}{l}
\text { there exists } H_{0} \subset \mathbf{R}^{n}, \operatorname{dim} H_{0} \geqslant 4 k / 5, \text { such that } \\
A H_{0}=H_{0} \text { and }\left\|P_{H^{\perp}} T\right\|_{2} \geqslant\|x\|_{2} \text { for } x \in H_{0} .
\end{array}\right.
$$

We have the following "measure theoretic" statement.

Proposition 5.1. Let $T$ satisfy the condition (2A). Then, for every $K_{0}>0$,

$$
\mathscr{P}\left(\left\{\|T: B \rightarrow B\|>K_{0}\right\}\right)>1-\left(\frac{c_{0} K_{0} n^{2}}{k^{5 / 2}}\right)^{k m / 2} .
$$

Moreover,

$$
\mathscr{P}\left(\left\{\begin{array}{l}
\text { there exists } Z, Z \perp\left[g_{i}, A g_{j}\right]_{i, j \leqslant m} \\
\text { such that, for some } j \leqslant k, P_{Z} T g_{j} \in K_{0} P_{Z} B
\end{array}\right\}\right)>1-\left(\frac{c_{0} K_{0} n^{2}}{k^{5 / 2}}\right)^{k m / 2} .
$$

$c_{0}$ is in both cases a numerical constant.

Proof. Clearly it is enough to prove the second statement. The argument is in the spirit of the proof of Fact 5.2 from [9]. We need the following lemma, which is a very slight modification of Claim 6.2 from [9] (the same proof works and so we omit it).

LEMMA 5.2. Let $\varepsilon, \sigma, K_{0}>0$ and let $W: H \rightarrow \tilde{H}(H, \tilde{H}$ are Hilbert spaces, $\operatorname{dim} H=k$ ) be such that $\#\left\{j: s_{j}(W) \geqslant 1\right\} \geqslant k_{0}$. Let $\tilde{B} \subset \tilde{H}$ be of the form $\tilde{B}=\operatorname{ac}\left\{y_{1}, y_{2}, \ldots, y_{M}\right\},\left\|y_{j}\right\|_{2} \leqslant 1$ for $j \leqslant M$, and let $g$ be an r.v. with distribution $N\left(0, \sigma^{2}, H\right)$. Then

$$
\mathscr{P}\left(\left\{W g \in K_{0} \tilde{B}\right\}\right) \leqslant\left(\frac{c K_{0} M \sqrt{k}}{k_{0}^{2} \cdot \sigma}\right)^{k_{0}} .
$$

Let us denote $G_{0}=\left[g_{i}\right]_{i \leqslant m}, \quad g_{j}=P_{H} g_{j}+P_{H^{\perp}} g_{j}=g_{j}^{\prime}+g_{j}^{\prime \prime}, G_{0}^{\prime \prime}=P_{H^{\perp}} G_{0}=$ $\left[g_{j}^{\prime \prime}\right]_{j \leqslant m}$ and $G^{\prime \prime}=G_{0}^{\prime \prime}+A G_{0}^{\prime \prime}=\left[g_{i}^{\prime \prime}, A g_{j}^{\prime \prime}\right]_{i, j \leqslant m}$ (note that since $A H=H$ and hence also $A H^{\perp}=H^{\perp}$, both $P_{H}$ and $P_{H^{\perp}}$ commute with $A$ and so e.g. $P_{H^{\perp}} A g_{j}=$ $\left.A P_{H^{\perp}} g_{j}=A g_{j}^{\prime \prime}\right)$. Finally, choose $Z=\left(G^{\prime \prime}+P_{H^{\perp}} T G_{0}^{\prime \prime}\right)^{\perp} \cap H^{\perp}$. Observe that

$1^{\circ} g_{1}^{\prime}, \ldots, g_{m}^{\prime}, g_{1}^{\prime \prime}, \ldots, g_{m}^{\prime \prime}$ are independent r.v.'s with distribution $N(0, k / n, H)$ for $g_{j}^{\prime}$ 's and $N\left(0,(n-k) / n, H^{\perp}\right)$ for $g_{j}^{\prime \prime}$ 's.

$2^{\circ} Z \subset H^{\perp}$, codimension of $Z$ in $H^{\perp}$ is $\leqslant 3 m \leqslant 3 k / 10 ; Z \perp G^{\prime \prime}=\left[g_{i}^{\prime \prime}, A_{g j}^{\prime \prime}\right]$.

$3^{\circ} G^{\prime \prime}$ and hence $Z$ depend only on $g_{j}^{\prime \prime}$ 's and so are independent of $g_{j}^{\prime \prime}$.

Consider now $T_{0} \stackrel{\text { df }}{=} P_{Z} T=P_{Z} P_{H^{\perp}} T$. As $\operatorname{rank}\left(T_{0}-P_{H^{\perp}} T\right) \leqslant 3 k / 10$ by $2^{\circ}$, we have (cf. (2A))

$$
\left\{\begin{array}{l}
\text { there is } H_{0} \subset H, \operatorname{dim} H_{0}=k_{0} \geqslant k / 2, \text { such that } \\
\left\|T_{0} x\right\|_{2} \geqslant\|x\|_{2} \text { for } x \in H_{0} .
\end{array}\right.
$$

By $3^{\circ}, H_{0}$ depends only on $g_{j}^{\prime \prime}$ 's and so is independent of $g_{j}^{\prime \prime}$ s.

We are now ready to estimate the probability in the assertion of Proposition 5.1. We have

$$
\begin{aligned}
\|T: B \rightarrow B\| \leqslant K_{0} & \Rightarrow T g_{j} \in K_{0} B \text { for } j=1,2, \ldots, m \\
& \Rightarrow P_{H^{\perp}} T g_{j} \in K_{0} P_{H^{\perp}} B \Leftrightarrow P_{H^{\perp}} T g_{j}^{\prime}+P_{H^{\perp}} T g_{j}^{\prime \prime} \in K_{0} P_{H^{\perp}} B \\
& \Rightarrow P_{Z} P_{H^{\perp}} T g_{j}^{\prime}+P_{Z} P_{H^{\perp}} T g_{j}^{\prime \prime}=T_{0} g_{j}^{\prime}+0 \in K_{0} P_{Z} P_{H^{\perp}} B
\end{aligned}
$$


and

$$
\begin{aligned}
P_{Z} P_{H^{\perp}} B & =P_{Z}\left(\operatorname{ac}\left\{P_{H^{\perp}} e_{i}, g_{j}^{\prime \prime}, A g_{h}^{\prime \prime}\right\}_{i \leqslant n ; j, h \leqslant m}\right) \\
& =\operatorname{ac}\left\{P_{Z} e_{i}, P_{Z} g_{j}^{\prime \prime}, P_{Z} A g_{h}^{\prime \prime}\right\}=\operatorname{ac}\left\{P_{Z} e_{i}\right\}=P_{Z} B_{1}^{n} .
\end{aligned}
$$

Thus $W=T_{0 \mid H}$ satisfies the assumptions of Lemma 5.2 with $k_{0} \geqslant k / 2$ (cf. (3)), $M=n, \tilde{B}=P_{Z} B=P_{Z} B_{1}^{n}=\operatorname{ac}\left\{P_{Z} e_{i}\right\}_{i \leqslant n}$ and $\sigma^{2}=k / n\left(\right.$ see $\left.3^{\circ}\right)$. Therefore

$$
\begin{aligned}
\mathscr{P}(\{\| T: B & \left.\left.\rightarrow B \| \leqslant K_{0}\right\}\right) \leqslant \mathscr{P}\left(\left\{T_{0} g_{j}^{\prime} \in K_{0} P_{Z} B_{1}^{n}, j=1,2, \ldots, m\right\}\right) \\
& =\left[\mathscr{P}\left(\left\{T_{0} g_{1}^{\prime} \in K_{0} P_{Z} B_{1}^{n}\right\}\right)\right]^{m} \leqslant\left(\frac{c K_{0} n \sqrt{k}}{k_{0}^{2} \cdot k / n}\right)^{k_{0} m} \\
& =\left(\frac{c K_{0} n^{2}}{k_{0}^{2} \sqrt{k}}\right)^{k_{0} m} \leqslant\left(\frac{4 c K_{0} n^{2}}{k^{5 / 2}}\right)^{k m / 2}
\end{aligned}
$$

and hence the assertion of Proposition 5.1 follows with $c_{0}=4 c$.

Q.E.D.

(c) The " $\varepsilon$-net" argument. Assume for simplicity that $k(\geqslant \delta n)$ is a multiple of 10 and choose $m=k / 10$. Then the statement in the assertion of Proposition 5.1 becomes

$$
\mathscr{P}\left(\left\{\|T: B \rightarrow B\|>K_{0}\right\}\right)>1-\left(\frac{c_{0} K_{0}}{\delta^{5 / 2} \sqrt{n}}\right)^{\delta^{2} n^{2} / 20} .
$$

Fix $K>0$ (to be specified later) and define

$$
\mathscr{U}=\left\{T \in \mathrm{GL}(n):\left\|T: l_{1}^{n} \rightarrow l_{1}^{n}\right\| \leqslant 2 K, T \text { satisfies }(2 A)\right\} .
$$

By Fact 5.1 of [9] (essentially due to Gluskin), for any $\varepsilon \in(0,1), \mathscr{U}$ admits an $\varepsilon \cdot 2 K / \sqrt{n}=$ net (in the operator norm on $l_{2}^{n}$ ), say $\mathscr{N}_{\varepsilon}$, such that $\# \mathscr{N}_{\varepsilon} \leqslant\left(C_{0} / \varepsilon\right)^{n^{2}}$. We need this fact just for $\varepsilon=\frac{1}{4}$, so denote by $\mathscr{N}=\mathscr{N}_{1 / 4}$ the $k / 2 \sqrt{n}$-net, $\# \mathscr{N} \leqslant C_{1}^{n^{2}}$. It follows from Proposition 5.1 (cf. (4)) applied with $K_{0}=2 K$ that if $K \leqslant c_{2} \sqrt{n}$ (where $c_{2}=c_{2}(\delta)=\delta^{5 / 2}\left(2 c_{0}\right)^{-1}\left(2 C_{1}\right)^{-20 / \delta^{2}}$ ), then

$$
C_{1}^{n^{2}} \cdot\left(\frac{c_{0} K_{0}}{\delta^{5 / 2} \sqrt{n}}\right)^{\delta^{2} n^{2} / 20} \leqslant\left(\frac{1}{2}\right)^{n^{2}}
$$

and hence with probability $\geqslant 1-\left(\frac{1}{2}\right)^{n^{2}}$ we have $\|T: B \rightarrow B\|>2 K$ and moreover

$$
\left\{\begin{array}{l}
\text { there exists } Z \perp\left[g_{i}, A g_{j}\right]_{i, j \leqslant m} \\
\text { such that } P_{Z} T g_{j} \notin 2 K P_{Z} B \text { for some } j \leqslant m
\end{array}\right.
$$

for every $T \in \mathscr{N}$. Consider now any $B$ satisfying (5) and additionally

$$
\left\|g_{j}\right\|_{2} \leqslant 2 \text { for } j=1,2, \ldots, m \text {. }
$$

Note that $\mathscr{P}(\{(6)\}) \geqslant 1-\alpha^{n}$ for some numerical $\alpha<1((\mathrm{i}), \S 2)$. We claim that any such $B$ verifies the assertion of Theorem 1.4 with $c=c_{2}(\delta)$ as above (except that, in view of (6), we have only $\frac{1}{2}\|\cdot\|_{2} \leqslant\|\cdot\|_{B}$; replacing $\|\cdot\|_{B}$ by $\|\cdot\|_{2} \vee\|\cdot\|_{B}$ or each $g_{j}$ by $\frac{1}{2} g_{j}$ takes care of that problem). To show this assume the opposite is true. Let, 
for some $B$ satisfying (5) and (6), there exist $T$ satisfying (2) such that

$$
\|T: B \rightarrow B\| \leqslant K \text {. }
$$

By an argument identical to that in the proof of Proposition 4.1 of [9] it then follows that there exists $\tilde{T},\left\|\tilde{T}: l_{1}^{n} \rightarrow l_{1}^{n}\right\| \leqslant K<2 K$, such that if, as before, $G=$ $\left[g_{i}, A g_{j}\right]_{i, j \leqslant m}$ and $Q=P_{G^{\perp}}$, then

$$
Q T=Q \tilde{T}
$$

In particular, $\operatorname{rank}(T-\tilde{T}) \leqslant \operatorname{dim} G \leqslant 2 m \leqslant k / 5$ and hence $\tilde{T}$ satisfies the condition (2A) (note that $A G^{\perp}=G^{\perp}$ ) and so $\tilde{T} \in \mathscr{U}$. Therefore there exists $T_{1} \in \mathscr{N}$ such that $\left\|\tilde{T}-T_{1}\right\|_{l_{2}^{n} \rightarrow l_{2}^{n}} \leqslant K / 2 \sqrt{n}$. By assumption (5) on $B$ this shows that there is a $Z \perp G$ and $j \in\{1,2, \ldots, m\}$ such that

$$
P_{Z} T_{1} g_{j} \notin 2 K P_{Z} B
$$

On the other hand, since obviously

$$
\left\|\tilde{T} g_{j}-T_{1} g_{j}\right\|_{2} \leqslant\left\|\tilde{T}-T_{1}\right\|_{l_{2}^{n} \rightarrow l_{2}^{n}}\left\|g_{j}\right\|_{2} \leqslant \frac{K}{2 \sqrt{n}} \cdot 2=\frac{K}{\sqrt{n}}
$$

(we used (6)), we have

$$
\tilde{T} g_{j}-T_{1} g_{j} \in \frac{K}{\sqrt{n}} B_{2}^{n} \subset K B_{1}^{n} \subset K B
$$

and hence

$$
P_{Z} T g_{j}-P_{Z} T_{1} g_{j} \in K P_{Z} B
$$

Now (9) and (10) together imply that $P_{Z} \tilde{T} g_{j} \notin K P_{Z} B$ and, a fortiori, since $P_{Z} Q=P_{Z}$ and $Q T=Q \tilde{T}$ by (8), $Q T g_{j}=Q \tilde{T} g_{j} \notin K Q B$, which contradicts (7).

This concludes the proof of Theorem 1.4.

Sketch of the proof of Theorem 1.4A. In the case of trivial $\Gamma$ one essentially follows [4]: to construct the space one adds $m$ random points to the ball of $l_{1}^{n}$, this time with any $m$ such that $C n(\ln n)^{2} \leqslant m \leqslant n^{2}$ for an appropriate numerical constant $C$. The "measure theoretic" part of the argument-based on an analogue of Lemma 4 from [4] rather than on Lemma 5.2-is much less complicated than in the case of Theorem 1.4 since we do not (and cannot) annihilate the space $Z^{\perp}$ (we pay for this with a weaker estimate). The only source of apparent difficulty is the presence of the terms $P_{H^{\perp}} T g_{j}^{\prime \prime}$, but they can be handled using the fact that the densities of measures $N\left(0, \sigma^{2}, l_{2}^{r}\right)$ are symmetric and radially decreasing. The " $\varepsilon$-net" part can be taken care of as in [4]: note that, for fixed $k$, one only needs to find a net for the set $\left\{P_{H^{\perp}} T: T\right.$ satisfies $\left.\left(M_{k, 1}\right),\left\|T: l_{2}^{n} \rightarrow l_{2}^{n}\right\| \leqslant n, H \in G_{k, n}\right\}$ of rank $k$ operators. The case of general $\Gamma$ requires only obvious modifications.

6. Proof of Theorem 1.5. Theorem 1.5 will follow immediately from Theorem 1.4 and the following

Proposition 6.1. Let $\delta>0$ and let $T: \mathbf{R}^{n} \rightarrow \mathbf{R}^{n}$ be such that, for any $\lambda \in \mathbf{R}$, $\|T-\lambda I\|_{C_{0}} \geqslant \delta n$. Then $T$ satisfies the mixing condition $\left(M_{k, \alpha}\right)$ with some $k \geqslant \delta n / 36$ and $\alpha=\delta / 6$. 
Indeed, suppose we have proved Proposition 6.1. Apply the first part of Theorem 1.4 with $\delta / 36$ instead of $\delta$. This gives $B=\left(\mathbf{R}^{n},\|\cdot\|_{B}\right)$ with $\|\cdot\|_{2} \leqslant\|\cdot\|_{B} \leqslant\|\cdot\|_{1}$ satisfying, by Proposition 6.1, the first assertion of Theorem 1.5 (about norms of operators). The facts that $B$ can be chosen so that the cotype 2 constant of $B$ does not exceed some numerical constant $c_{1}$ and that $B$ satisfies the Grothendieck theorem with constant $c_{1}$ follow from the "random" construction of $B-\operatorname{see} \$ 5(\mathrm{a})$.

REMARK 6.2. It should be noted that the following converse to Proposition 6.1 holds: If $T: l_{2}^{n} \rightarrow l_{2}^{n}$ verifies $\left(M_{k, \alpha}\right)$, then, for any $\lambda \in \mathbf{R},\|T-\lambda I\|_{C_{0}} \geqslant k \alpha /(1+\alpha)$.

For the proof of Proposition 6.1 we need the following simple variant of Lemma 4.1.

LeMmA 6.3. Given $T: l_{2}^{n} \rightarrow l_{2}^{w}$ and $G \subset l_{2}^{n}$, $\operatorname{dim} G=p$, there exists an orthonormal sequence $f_{1}, f_{2}, \ldots, f_{k}$ in $G$ with $k \geqslant p / 4$ such that

$$
\left(T f_{i}, T f_{j}\right)=\left(T f_{i}, f_{j}\right)=0 \text { if } 1 \leqslant i, j \leqslant k, i \neq j .
$$

Proof. Argue as in Lemma 4.1 omitting all references to $A$ and $s$-numbers of $T$.

Proof of Proposition 6.1. Observe first that it is enough to exhibit an orthonormal sequence $h_{1}, h_{2}, \ldots, h_{k}, k \geqslant \delta n / 36$, satisfying

(I) $\left(T h_{i}, T h_{j}\right)=\left(T h_{i}, h_{j}\right)=0$ for $i \neq j, i \leqslant i, j \leqslant k$,

(II) $\left\|P_{\left[h_{j}\right]^{\perp}} T h_{j}\right\|_{2} \geqslant \delta / 6$ for $j \leqslant k$.

Indeed, then the space $H=\left[h_{1}, h_{2}, \ldots, h_{k}\right]$ works: just observe that, for each $j$, $P_{H^{\perp}} T h_{j}=P_{\left[h_{j}\right]^{\perp}} T h_{j}$ and the sequence $\left(P_{H^{\perp}} T h_{j}\right)_{j \leqslant k}$ is orthogonal.

To construct $\left(h_{j}\right)$ we proceed as follows. Let $T=\sum_{j=1}^{n} \lambda_{j}\left(u_{j}, \cdot\right) \tilde{u}_{j}$ be the polar decomposition of $T$. Since $\|T\|_{C_{0}}=\|T-O I\|_{C_{0}} \geqslant \delta n$, we must have

$$
\#\left\{j: \lambda_{j} \geqslant 5 \delta / 6\right\} \geqslant \delta n / 6
$$

or, in other words, if $m$ is the smallest integer greater than or equal to $\delta n / 6$, then $\lambda_{m} \geqslant 5 \delta / 6\left(\left(\lambda_{j}\right)\right.$ is assumed to be nondecreasing $)$. Let us consider two cases:

(i) $\lambda_{m}-\lambda_{n-m+1}<\delta / 3$,

(ii) $\lambda_{m}-\lambda_{n-m+1} \geqslant \delta / 3$.

Case (i). Note that if $\rho=\lambda_{m}-\delta / 6(\geqslant 2 \delta / 3)$ and $m \leqslant j \leqslant n-m+1$, then $\left|\rho-\lambda_{j}\right| \leqslant \delta / 6$. Thus if $F=\left[u_{j}\right]_{m \leqslant j \leqslant n-m+1}$, then

$$
\operatorname{codim} F=2 m-2<\delta n / 3
$$

and if $x \in F$, then

$$
(\rho-\delta / 6)\|x\|_{2} \leqslant\|T x\|_{2} \leqslant(\rho+\delta / 6)\|x\|_{2} .
$$

For $x \in \mathbf{R}^{n}$ consider the condition

$$
\|T x-\lambda x\|_{2} \leqslant \frac{\delta}{3}\|x\|_{2}
$$

for $\lambda=\rho$ and $\lambda=-\rho$; we will also denote by $\sim(13)_{\lambda}$ the reverse (nonstrict) inequality. Our present goal is to prove the following

Claim. At least one of the following statements holds.

(A) There exists $E_{0} \subset F, \operatorname{dim} E_{0} \geqslant \delta n / 9$, such that every $x \in E_{0}$ verifies both $\sim(13)_{\rho}$ and $\sim(13)_{-\rho}$.

(B) There exist $E_{1}, E_{2} \subset F$ with $E_{1} \perp E_{2}$ and $\operatorname{dim} E_{1}=\operatorname{dim} E_{2} \geqslant \delta n / 9$ such that every $x \in E_{1}$ verifies (13) $)_{-\rho}$ and every $x \in E_{2}$ verifies (13) $)_{\rho}$. 
Proof. Observe first that since $\|T \pm \rho I\|_{C_{0}} \geqslant \delta n$, (11) implies that $\left\|T_{\mid F} \pm \rho I_{F}\right\|_{C_{0}}$ $\geqslant \frac{2}{3} \delta n$. Therefore there exist $F_{1}, F_{2} \subset F, \operatorname{dim} F_{i} \geqslant \delta n / 3$ such that every $x \in F_{1}$ satisfies $\sim(13)_{-\rho}$ and every $x \in F_{2}$ satisfies $\sim(13)_{\rho}$. If no $E_{0}$ satisfying the condition (A) exists, then it follows from the remarks following the definition of polar decomposition in $\S 2$, applied to the operator $T_{\mid F_{1}}+\rho I_{F_{1}}$ (resp. $T_{\mid F_{2}}-\rho I_{F_{2}}$ ), that there exists $F_{1}^{\prime} \subset F_{1}, \operatorname{dim} F_{1}^{\prime} \geqslant 2 \delta n / 9$ (resp. $\left.F_{2}^{\prime} \subset F_{2}, \operatorname{dim} F_{2}^{\prime} \geqslant 2 \delta n / 9\right)$ such

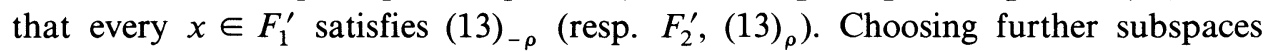
$E_{i} \subset F_{i}^{\prime}$ with $\operatorname{dim} E_{i} \geqslant \delta n / 9$ and $E_{1} \perp E_{2}$ concludes the proof of the claim.

To settle case (i) it remains to deduce the assertion of Proposition 6.1 from the Claim. To this end, we observe that if $E$ is either $E_{0}$ (from (A)) or the graph of any isometry from $E_{1}$ onto $E_{2}\left(E_{1}, E_{2}\right.$ as in (B)), then

$$
\left\|P_{[x]^{\perp}} T x\right\|_{2} \geqslant \frac{\delta}{6}\|x\|_{2} \text { for } x \in E .
$$

A simple geometric argument (the reader is advised to draw pictures) works in both cases. If $x \in E_{0}$ with $\|x\|_{2}=1$ and $T x=a x+x^{\prime}$ with $x^{\prime} \perp x$, then it is easily shown that (12), $\rho \geqslant \frac{2}{3} \delta$, and $\sim(13)_{\rho \cdot \operatorname{sgn} a}$ show that $\left\|x^{\prime}\right\|_{2}>\delta / 6$. On the other hand, if $y \in E_{1}$ and $z \in E_{2}$ are such that $\|y\|_{2}=\|z\|_{2}=1$, then $\|T y+\rho y\|_{2} \leqslant \delta / 3$, $\|T z-\rho z\| \leqslant \delta / 3$ and so if $x=2^{-1 / 2}(y+z)$ and $x^{\prime}=2^{-1 / 2}(y-z)$, then $x^{\prime} \perp x$, $\|x\|_{2}=\left\|x^{\prime}\right\|_{2}=1$ and $\left(T x, x^{\prime}\right) \geqslant \frac{1}{3}(2-\sqrt{2})>\frac{1}{6}$ (we also use $\rho \geqslant \frac{2}{3} \delta$ here). This establishes (14).

Finally notice that in either case $E$ satisfying (14) also verifies $\operatorname{dim} E \geqslant \delta n / 9$. Therefore applying Lemma 6.3 to $E$ we get an orthonormal sequence satisfying (I) and (II), as required.

Case (ii). First apply Lemma 6.3 with $G=\left[u_{1}, \ldots, u_{m}\right]$ to obtain an orthonormal sequence $f_{1}, \ldots, f_{k}, k \geqslant m / 4$. Then define $q$ to be the smallest integer not less than $m / 6$ (note that $q \geqslant \delta n / 36$ and $E=\left[f_{1}, \ldots, f_{q}\right]$ ). Again apply Lemma 6.3 with $G=\left[u_{n-m+1}, \ldots, u_{n}\right] \cap(T E)^{\perp} \cap T^{-1}\left(E^{\perp}\right)$ to obtain $E^{\prime}=\left[f_{1}^{\prime}, \ldots, f_{q}^{\prime}\right]$; then $E^{\prime} \perp$ $T E$ and $T E^{\prime} \perp E$ (observe that we have automatically $E \perp E^{\prime}$ and $T E \perp T E^{\prime}$ since $u_{j}$ 's came from the polar decomposition of $T$ ).

Now set $h_{j}=2^{-1 / 2}\left(f_{j}+f_{j}^{\prime}\right)$ for $j=1,2, \ldots, q$. We claim that these $h_{j}$ 's satisfy the conditions (I) and (II). Indeed, (I) is clear since $f_{j}$ 's and $f_{j}^{\prime}$ 's were obtained via Lemma 6.3 and since $E, E^{\prime}, T E, T E^{\prime}$ are mutually orthogonal. To show (II), we observe that

$$
\begin{aligned}
\left\|P_{\left[h_{j}\right]^{\perp}} T h_{j}\right\|_{2}^{2} & =\frac{1}{2}\left\|T\left(f_{j}+f_{j}^{\prime}\right)\right\|_{2}^{2}-\frac{1}{4}\left|\left(T\left(f_{j}+f_{j}^{\prime}\right),\left(f_{j}+f_{j}^{\prime}\right)\right)\right|^{2} \\
& \geqslant \frac{1}{4}\left(\left\|T f_{j}\right\|-\left\|T f_{j}^{\prime}\right\|\right)^{2} \geqslant \frac{1}{4}\left(\lambda_{m}-\lambda_{n-m+1}\right)^{2} \geqslant(\delta / 6)^{2}
\end{aligned}
$$

by (ii). This concludes the proof of Proposition 6.1.

Proof of THEOREM 1.5A. We use Theorem 1.4A and the following

Lemma 6.4. Let $T: \mathbf{R}^{n} \rightarrow \mathbf{R}^{n}$ and denote $\kappa=\inf _{\lambda \in \mathbf{R}}\|T-\lambda I\|_{w-C_{1}}$. Then there exist $k, \alpha$ with $k \alpha \geqslant c \kappa$ ( $c$ a universal constant) such that $T$ satisfies the condition $\left(M_{k, \alpha}\right)$. 
The proof of Lemma 6.4 goes along the same lines as the proof of Proposition 6.1. First, by replacing $T$ by $T-\lambda_{0} I$ for appropriate $\lambda_{0}$, we may assume that $\|T\|_{w-C_{1}}=\kappa$. Assume, for simplicity, that $n$ is even and set $\rho=s_{n / 2}(T)$. Consider now the operator $T-\rho I$ and $\|T-\rho I\|_{w-C_{1}}=m_{1} \cdot s_{m_{1}}(T-\rho I)(\geqslant \kappa)$, denote $\beta_{1}=s_{m_{1}}(T-\rho I)$. Assume first that $m_{1} \leqslant n / 2$ and consider two cases:

(i) $s_{m_{1}}(T)-s_{n-m_{1}+1}<\frac{1}{2} \beta_{1}$,

(ii) $s_{m_{1}}(T)-s_{n-m_{1}+1} \geqslant \frac{1}{2} \beta_{1}$.

In case (ii) ${ }^{\prime}$ we argue similarly as in case (ii) of Proposition 6.1. In case (i)' notice that we must necessarily have

$$
\beta_{1}=s_{m_{1}}(T-\rho I) \leqslant s_{m_{1}}(T)+\rho<\rho+\frac{1}{2} \beta_{1}+\rho
$$

and so $\rho>\frac{1}{4} \beta_{1}$. Therefore $m_{1} \beta_{1} \geqslant \kappa \geqslant \frac{1}{2} n \cdot \rho>\frac{1}{2} n \cdot \frac{1}{4} \beta_{1}$ and hence $m_{1} \geqslant \frac{1}{8} n$ (the case $m_{1}>\frac{1}{2} n$ also falls in here). Considering similarly $T+\rho I$ we get that the only unsettled case is when $\|T+\rho I\|_{w-C_{1}}=m_{2} \beta_{2}$ etc., with $m_{2} \geqslant \frac{1}{8} n$. But this type of situation is covered by Proposition 6.1. Thus Lemma 6.4- and hence Theorem 1.5A -is proved.

\section{REFERENCES}

1. J. Bourgain, A complex space $X$ such that $X$ and $\bar{X}$ are not isomorphic, Proc. Amer. Math. Soc. (to appear).

2. T. Figiel, S. Kwapién and A. Pelczyński, Sharp estimates for constants of local unconditional structure for Minkowski spaces, Bull. Acad. Polon. Sci 25 (1977), 1221-1226.

3. E. D. Gluskin, The diameter of the Minkowski compactum is roughly equal to $n$, Functional Anal. Appl. 15 (1981), 72-73. (Russian)

4. Finite dimensional analogues of spaces without a basis, Dokl. Akad. Nauk SSSR 261 (1981), 1046-1050. (Russian)

5. N. J. Kalton, Informal communication.

6. J. Lindenstrauss and L. Tzafriri, Classical Banach spaces. I, Springer-Verlag, 1977.

7. P. Mankiewicz, Finite dimensional Banach spaces with symmetry constant of order $\sqrt{n}$, Studia Math. 79 (1984), 193--200.

8. Informal communication.

9. S. J. Szarek, The finite dimensional basis problem with an appendix on nets of Grassmann manifolds, Acta Math. 151 (1983), 153-179.

Department of Mathematics, Case Western Reserve University, Cleveland, Ohio 44106 